\title{
Effect of Decontamination Procedures on Marginal and Internal Adaptation in Saliva Contaminated Resin Composite Restorations
}

Eftychia Pappa ( $\nabla$ effiepappa84@yahoo.com )

National and Kapodistrian University of Athens https://orcid.org/0000-0002-1631-9871

Konstantinos Masouras

National and Kapodistrian University of Athens

Vasileios Margaritis

Walden University

Afrodite Kakaboura

National and Kapodistrian University of Athens

Research article

Keywords: saliva, marginal adaptation, adhesive, microgap, contamination

Posted Date: March 4th, 2020

DOl: https://doi.org/10.21203/rs.3.rs-16038/v1

License: (c) (i) This work is licensed under a Creative Commons Attribution 4.0 International License.

Read Full License 


\section{Abstract}

Background: The aim of this study was to evaluate the effect of natural saliva contamination of dentin, on marginal and internal adaptation of resin-composite restorations performed with an etch-and-rinse adhesive and determine the efficiency of various decontamination techniques. Methods: A hundred and twenty human molars and premolars were randomly distributed into six groups $(n=20)$. Enamel was removed and standardized dentin cavities (diameter: $3 \mathrm{~mm}$, depth: $2 \mathrm{~mm}$ ) were filled with a two-step etchand-rinse adhesive and resin-composite under six different surface treatments: (1) adhesive application following manufacturers' instructions (control); (2) dentin etching, 5-s saliva, 5-s air-dry, adhesive; (3) dentin etching, 5-s saliva, 10-s water-rinse, 5-s air-dry, adhesive; (4) adhesive application/light-cure, 5-s saliva, 5-s air-dry; (5) adhesive application/light-cure, 5-s saliva, 10-s water-rinse, 5-s air-dry; (6) adhesive application/light-cure, 5-s saliva, 10-s water-rinse, 5-s air-dry, adhesive reapplication. Wall adaptation was evaluated at the upper surface and at two consecutive depth levels of $0.5 \mathrm{~mm}$ each, by measuring the length of the debonded margins relative to the cavity periphery (\%DM) and the width of the maximum marginal gap (MG). Results: Statistically significant differences occurred among groups, presenting inferior adaptation after contamination took place. Group (1) at the upper surface presented the most satisfactory adaptation, whereas, groups (4) and (5) had the significantly poorest adaptation in all surfaces. Groups (2), (3), and (6) revealed no statistically significant differences in comparison to group (1). Conclusion: Saliva contamination resulted in deterioration of marginal and internal adaptation when it occurred after the adhesive application. Reapplication of the adhesive restored DM and MG values.

\section{Background}

A reliable and stable bond between resin-composite and tooth structure is needed in order to achieve an optimal clinical performance. The importance of moisture and contamination control is highlighted throughout the dental literature and all efforts are focused on keeping the adhesive substrate free of oral contaminants such as saliva, blood, intersulcular fluid and handpiece oil. Inadequate isolation of the operating field jeopardizes the restoration quality and longevity and can possibly lead to consequences such as microleakage, marginal discoloration, postoperative sensitivity, caries and pulpal irritation. [1]

The use of rubber dam is recommended for proper isolation and prevention of cavity contamination. Nevertheless, carious lesions are often located in areas where it is difficult to achieve proper moisture control, especially when the site is near or at the gingival margin. Clinicians often encounter difficulties in adequate isolation of the operating field in cases when the cavity margins are located in dentin such as the cervical margins of class $\mathrm{V}$ and class II restorations, in pediatric patients with poor cooperation and when seating indirect restorations with subgingival margins. [2]

The effect of enamel saliva contamination on the bond strength of adhesive systems has been previously investigated and studies concluded that oral fluids significantly reduce the bond strength. [3] Compared to enamel, the dentin structure is more complex and despite many investigations have evaluated the effect of saliva contamination on the adhesive interface, results are considered to be 
inconclusive. A number of studies reported a significant reduction of bond strength between dental adhesives and saliva-contaminated dentin substrate [4-6] whereas others found no significant differences between contaminated and non-contaminated dentin substrate. [7]

Shear and microtensile bond tests have been usually performed in order to demonstrate the effect of saliva contamination on the quality of adhesive bonding. [4, 7] Additionally, the effect of contamination on the longevity of bonded restorations has been evaluated through microleakage tests. [6] Despite the significant number of studies, there is no clear conclusion whether contamination negatively affects the adhesive interface or not. A variety of influencing factors seems to complicate the interpretation of the results. Such factors are the composition of adhesive systems, the duration of the contamination and the decontamination treatment of the substrate. Moreover, to the best of our knowledge, there is only one study on how saliva contamination affects the adaptation of adhesive restorations and the formation of microgaps in the adhesive interface. [7]

The aim of this study was to evaluate the effect of natural saliva contamination of dentin, on marginal and internal adaptation of resin-composite restorations performed with a two-step etch-and-rinse adhesive, before and after adhesive curing and also to determine the efficiency of various decontamination techniques. The null hypothesis tested was that the adaptation of resin-composite restorations on dentin walls would not be affected by saliva contamination or by the decontamination techniques applied.

\section{Methods}

The experimental protocol applied has been approved by the local Committee of Ethics according to the guidelines provided by the World Medical Association Declaration of Helsinki. Written informed consent was obtained from all participants whose teeth were used in the study. One hundred and twenty freshly extracted caries-free human molars and premolars were selected and stored at $4^{\circ} \mathrm{C}$ in $0.5 \%$ chloramine-T containing distilled water. The teeth were examined to reassure absence of cracks, fissures or any type of restoration. Afterwards, they were embedded in epoxy resin (Epofix, Struers, Denmark) and subsequently, occlusal enamel was removed with a low speed diamond saw under copious water cooling (Isomet, Buehler Ltd, Lake Bluff, IL, USA) and exposed dentin was ground wet with a 600 grit SiC paper.

Standardized cylindrical dentin cavities (diameter: $3 \mathrm{~mm}$, depth: $2 \mathrm{~mm}$ ) were prepared using a 3-mmdiameter diamond bur (Proxxon, Niersdorf, Germany) on a micro-milling machine (MF70 Micro-Miller, Proxxon, Niersdorf, Germany) under copious water cooling. Dentin cavities were examined under 200x magnification to ensure absence of pulp exposure. All cavities were filled with a two-step etch-and-rinse adhesive and resin-composite (Table 1, Fig. 1). The resin-composite was placed in a single layer, covered with a mylar strip, pressed against a transparent cover slip and photopolymerized for 40 seconds with a light-curing unit of $850 \mathrm{~mW} / \mathrm{cm}^{2}$ light intensity (Elipar Trilight 3M/ESPE, Seefeld, Germany). The restorations were ground gradually with a series of silicon carbide papers of 320-1000 grit size under wet 
conditions until exposure of the dentin cavity margins. Inspection was made after removing the surface debris with copious amounts of water.

Fresh natural saliva was collected near the time of contamination, provided by a single donor, after brushing, flossing and chewing a paraffin gum for $5 \mathrm{~min}$ prior to saliva collection. Saliva was collected in a sterile beaker and was used immediately. Each time, $15 \mu \mathrm{l}$ of saliva were collected using a micropipette and applied on the substrate. The saliva was left undisturbed for 5 seconds (s) before each decontamination technique was applied.

Teeth were randomly distributed into six groups of 20 each and a different surface treatment was accordingly applied:

- Group 1: adhesive application following manufacturers' instructions (control);

- Group 2: dentin etching, 5-s saliva, 5-s air-dry, adhesive;

- Group 3: dentin etching, 5-s saliva, 10-s water-rinse, 5-s air-dry, adhesive;

- Group 4: adhesive application/light-cure, 5-s saliva, 5-s air-dry;

- Group 5: adhesive application/light-cure, 5-s saliva, 10-s water-rinse, 5-s air-dry;

- Group 6: adhesive application/light-cure, 5-s saliva, 10-s water-rinse, 5-s air-dry, adhesive reapplication.

Wall adaptation was determined at the upper surface and at two consecutive depth levels of $0.5 \mathrm{~mm}$ each, after gradual grinding of each sample (Fig. 1); the dimensions were checked with a digital caliper. The specimens were observed under an optical microscope at 200x magnification and the parameters measured were the length of the debonded margins (DM) relative to the cavity periphery (\%DM) and the width of the maximum marginal gap (MG) (Fig. 2). The microscope used was ME 600 Eclipse (NikonKogakou, Japan) and the measurements were carried out by an image analysis software (Sigma Scan 4, Jandel Scientific, CA, USA). Images were recorded with parallel and crossed polarizers, to differentiate the presence of entrapped debris at the interface which might impose a bias on the results.

Since \%DM and MG values were not normally distributed, Kruskal-Wallis and Mann-Whitney U post-hoc tests were used to investigate the possible influence of saliva contamination and the different decontamination techniques before and after the adhesive polymerization on the marginal and internal adaptation. All reported probability values ( $p$-values) were compared to a significant level of $5 \%$. The analyses of coded data were carried out using IBM SPSS software version 21.0.

\section{Results}

Fig. 3 and 4 show representative images of restorations with perfect marginal adaptation and debonded margins, respectively. 
Results from the measurement of the length of the debonded margins relative to the cavity periphery (\%DM) and the width of the maximum marginal gap (MG) are summarized in Tables 2 and 3 , respectively.

Kruskal-Wallis test revealed statistically significant differences among the six groups, for both parameters tested at the 0.05 significance level. Mann-Whitney U multiple comparison tests were used to identify the relations among the experimental groups.

Group 1 was the only one that exhibited excellent marginal adaptation with absolute absence of microgaps at the upper surface of the restoration (level 1). However, no statistically significant differences were revealed between group 1 and each of groups 2, 3 and 6 for \%DM and MG values per depth level. Concerning the comparison among groups 2, 3 and 6, no differences were noticed for all recordings. Groups 4 and 5 showed statistically the highest \%DM and MG values, at all three depth levels, without differences between them.

\section{Discussion}

A gap-free restoration is considered as a key factor for the optimal clinical performance of resincomposite restorations. [8-12] Despite the fact that there is no threshold gap-size for the occurrence of secondary caries, the presence of marginal defects provides potential pathways for bacteria penetration and as a consequence, jeopardizes longevity and clinical success of adhesive restorations. [13]

During polymerization shrinkage, tension is expected in the adhesive interface and the resulting stress may cause deflection of dentin walls and/or bond failure with subsequent gap formation. In the present study, resin-composite was placed in a single increment of $2 \mathrm{~mm}$. A $2 \mathrm{~mm}$ of incremental application and subsequent polymerization is usually advised to ensure a full depth of cure and to reduce the net effect of polymerization shrinkage. $[13,14]$ Numerous factors are involved to the debonding phenomenon either during bond development or during the oral service of a restoration. Although several studies [4-7] have previously investigated the effect of saliva contamination on the adhesive interface, there is no sufficient evidence regarding the effect of saliva contamination on the adaptation of adhesive restorations. Parameters such as the extent of the debonded margin and the width of the marginal gap determine the quality of the adhesive interface and the ability of the adhesive system to prevent marginal debonding. [14] The marginal adaptation of the restorations is not necessarily similar to the internal adaptation to the cavity walls; therefore, in the present study, the internal adaptation was additionally evaluated as a factor with crucial contribution to the long term good clinical performance of the restorations.[12] In the current study, the cavity configuration factor was the same for all samples and only one adhesive system and resin-composite were used, to avoid interference by the aforementioned factors. Consequently, differences could be attributed to the different stages of contamination and decontamination techniques applied.

As already stated, the vast majority of previous studies on saliva contamination evaluate the effect of contamination on bond strength. [4-7] In the present study, marginal adaptation and gap formation were 
evaluated in order to obtain information concerning the sealing ability of the adhesive system on the contaminated dentin. Under the clinical point of view, saliva contamination is possible to occur either after the etching procedure and before the adhesive application or after the completion of the adhesive placement. Both conditions were investigated in the current study. As an experimental procedure, marginal and internal adaptation is considered to be laborious and time-consuming technique. It often yields false negative results and requires a large number of specimens in each group because of the high variability of the values. Despite the above limitations, it is thought to be more clinically relevant to evaluate the capability of an adhesive to maintain the tooth-restoration interface sealed, rather than to compare bond strength values. $[15,16]$

Direct optical microscopic observation of the specimens was preferred to evaluation of replicas by a scanning electron microscopy (SEM) since the first method allows measurements directly on dentin specimens instead of replicas, providing reliable phase identification and allowing evaluation of the same specimen at different levels. It is a simpler, less time-consuming and less destructive method, which avoids any potential drawbacks of the replica technique. [5]

In this study, human saliva was chosen as the contaminant, in order to achieve clinically relevant conditions, contrary to artificial saliva and substitutes which were used in other studies. $[17,18]$ SEM observations have shown that high-molecular-weight proteins diffuse into dentin tubules, altering the surface characteristics and competing with hydrophilic monomers during the hybridization process. [5, $19,20]$ In order to standardize the contaminating factor and avoid extreme changes in $\mathrm{pH}$ and buffering capacity, saliva was always collected from the same individual, following a typical oral hygiene protocol as previously described in Methods. In a study of Neelagiri et al., fresh whole saliva is considered an acceptable material to be used for contaminating testing as artificial saliva, which is deprived of macromolecules, may confound the results. [21]

Excite $\mathrm{F}$ is acetone-free, containing ethanol as a solvent. Acetone-containing adhesives are effective only on moist dentin. Water-based adhesives are insensitive to the degree of dentin moisture, but the adhesive layer must be adequately dried to remove water. On the other hand, ethanol combines the favorable properties of acetone and water and acts as a "water chaser", displacing water, carrying the resin inside dentin tubules. Ethanol may also denature the glycoprotein of the contaminated surface and clean the substrate. $[7,15]$

Saliva was left undisturbed for 5 seconds prior to the application of any decontamination technique, with the hypothesis that this is an average time for the clinician to notice and deal with the contamination. The time span during which the contaminant interacts with the dentin surface is of significant importance, as longer contamination time results in lower bond strengths than drying the saliva quickly. [22] This observation was related to evaporation of water and formation of a thick film of glycoprotein on the contaminated surfaces. The duration of contamination varies significantly among studies and it is a factor that should be particularly considered when comparing their findings. 
According to the results of the present study, the null hypothesis was rejected since both marginal and internal adaptation of resin-composite restorations on dentin walls were found to be negatively affected by the presence of saliva, when contamination took place after the adhesive application. Reapplication of adhesive restored adaptation and could be considered as the decontamination technique of choice. On the other hand, the saliva contamination that occurred after acid-etching and before adhesive application did not adversely affect the adaptation in a significant level. Air-drying for 5 seconds appears to be sufficient in such cases, with no need for water-rinse of the operation field.

An important outcome of the present study was that \%DM and MG values were not significantly affected when saliva contaminated the etched dentin and following removed either with air or with water. These findings are in agreement with other studies which showed that adhesion was not negatively affected when the duration of contamination was between 5 and 15 seconds. [5, 7] For extended contamination time (60 seconds), re-etching of the contaminated dentin effectively removes contamination, but it appears to also deteriorate the quality of adhesion due to the collapse of denatured collagen fibrils, increased thickness of the collagen layer and inadequate hybridization.[18, 23] As far as self-etch adhesive systems are concerned, they contain acidic monomers that promote adhesion to smear-covered dentin but the thick - due to proteinic complexes - smear layer may inhibit penetration of self- etching primers, thus lowering bond strength values in the contaminated substrate.[21, 23]

Contamination of the polymerized adhesive layer resulted in increased gap formation and deterioration of both marginal and internal adaptation. These results are consistent with the study of Fritz et al, where it was shown that contamination after light-curing of the adhesive reduced the shear bond strength of a two-step etch-and-rinse adhesive system to about $50 \%$ of control values. [24] It was hypothesized that the absorbed glycoproteins onto the poorly polymerized adhesive surface, inhibit adequate copolymerization. Another possible explanation suggests that rinsing and air-drying provides a collapsed layer of resin deprived collagen which cannot be effectively penetrated. [22, 23]

Conversely, several studies concluded that there was no adverse effect on bond strength values of twostep self-etching adhesives when contamination occurred after light-curing.[17, 25] This can be possibly explained by the simultaneous demineralization and infiltration achieved by these systems. Self-etching adhesives contain acidic monomers combined with hydrophilic monomers that simultaneously etch and prime dentin. Hydrophilic and acidic dentin adhesives are considered to be less sensitive to the presence of saliva and researchers have concluded that bond strength values to contaminated dentin are not negatively affected.[7, 19]

As far as the width of the formed gaps is concerned, mean values ranged between 0 and $30.74 \mu \mathrm{m}$, which are similar to the ones reported elsewhere.[10] At $0.5 \mathrm{~mm}$ in-depth level, groups 2 and 4 present particularly high SD values. At those groups, two extreme gaps were measured, which could be possibly attributed to false technique application. The presence of too much moisture on the substrate can create small, blister-like voids which appear like gaps in the interface and which have an impact on the 
performance of the adhesive material. Incomplete hybridization of the demineralised dentin leaves exposed collagen fibrils and causes nanoleakage through gaps of $20-100 \mathrm{~nm}$ size.[6]

In the present study, the performance of an etch-and-rinse adhesive system was evaluated in different stages of the adhesive procedure. It would be helpful to compare these findings with the adaptation obtained using a self-etch adhesive, to have a complete perception of adhesive systems' performance on contaminated substrates. Despite the fact that adaptation is considered to evaluate better the sealing ability of an adhesive system, it was also reported that the in vitro measured "marginal adaptation" had only a limited value in predicting the clinical performance of adhesives.[15] Evidently, the results of this in vitro study cannot be generalized to clinical practice. Measurements of adaptation were applied directly after the contamination; this, however, does not reflect the long term clinical performance of restorations and it should be taken into consideration that decontamination techniques and residual saliva could possibly accelerate the deterioration of the bond and consequently aggravate the adaptation.

\section{Conclusions}

Under the limitations of this study, the following conclusions can be drawn:

- Saliva contamination of etched dentin did not affect marginal and internal adaptation of the adhesive and resin-composite tested when the saliva was removed by air or water before the application of the adhesive.

- Inferior adaptation was observed in the contaminated dentin cavities when the contamination occurred after the adhesive application.

- Removal of saliva and reapplication of the adhesive restored the adaptation.

The findings of this study are of significant clinical relevance. The possible negative influence of saliva contamination after the adhesive application can be effectively reversed by reapplication of the adhesive.

\section{Abbreviations}

DM: debonded margins

\%DM: the length of the DM relative to the cavity periphery

MG: maximum marginal gap

SEM: scanning electron microscopy

\section{Declarations}

- $\quad$ Ethics approval and consent to participate: 
The experimental protocol applied has been approved by the local Committee of Ethics according to the guidelines provided by the World Medical Association Declaration of Helsinki. Written informed consent was obtained from all participants whose teeth were used in the study.

- $\quad$ Consent to publish:

Not Applicable

- $\quad$ Availability of data and materials:

The datasets analysed during the current study are presented in summary in Tables 1,2,3. Spreadsheets are available from the corresponding author on reasonable request.

- $\quad$ Competing interests:

None to declare. All authors declare no financial interest or conflict associated with this work.

- $\quad$ Funding:

Not Applicable

- $\quad$ Authors' Contributions:

All four authors meet the four criteria described by BMC Oral Health for authorship. EP, KM, VM and AK substantially contributed to the conception and design of the work; the analysis and interpretation of data; the revision of the draft and they all approved the final version of the manuscript to be published. This manuscript has been read and approved by all the authors. The requirements for authorship as stated earlier in this document have been met and each author believes that the manuscript represents honest work.

- $\quad$ Acknowledgments:

Not Applicable

\section{References}

1. Eiriksson SO, Pereira PN, Swift EJ et al. Effects of saliva contamination on resin-resin bond strength. Dent Mater 2004;20:37-44.

2. Auschill TM, Koch CA, Wolkewitz $M$ et al. Occurence and causing stimuli of postoperative sensitivity in composite restorations. Oper Dent. 2009;34:3-10.

3. Hickel R, Manhart J. Longevity of restorations in posterior teeth and reasons for failure. J Adhes Dent 2001;3:45-64.

4. El-Kalla IH, García-Godoy F. Saliva contamination and bond strength of single-bottle adhesives to enamel and dentin. Am J Dent 1997;10:83-7. 
5. Yazici AR, Tuncer D, Dayangaç $B$ et al. The effect of saliva contamination on microleakage of an etch-and-rinse and a self-etching adhesive. J Adhes Dent 2007;9:305-9.

6. Duarte SJ, Lolato AL, de Freitas CR et al. SEM analysis of internal adaptation of adhesive restorations after contamination with saliva. J Adhes Dent 2005;7:51-6.

7. Xie J, Powers JM, McGuckin RS. In vitro bond strength of two adhesives to enamel and dentin under normal and contaminated conditions. Dent Mater 1993;9:295-9.

8. Perdigão J. New developments in dental adhesion. Dent Clin North Am 2007;51:333-357.

9. Strydom C. Handling protocol of posterior composites Rubber Dam. SADJ 2005;60:292-293.

10. Ritter AV, Swift EJ Jr, Heymann HO et al. An eight-year clinical evaluation of filled and unfilled onebottle dental adhesives. J Am Dent Assoc 2009;140:28-37.

11. Park JW, Lee KC. The influence of salivary contamination on shear bond strength of dentin adhesive systems. Oper Dent 2004;29:437-42.

12. Tay FR, Gwinnett AJ, Pang KM et al. Variability in microleakage observed in a total-etch wet-bonding technique under different handling conditions. J Dent Res 1995;74:1168-78.

13. Feilzer AJ, De Gee AJ, Davidson CL. Setting stress in composite resin in relation to configuration of the restoration. J Dent Res 1987;66:1636-9.

14. Kaisarly D, Gezawi ME. Polymerizationshrinkageassessment of dental resin composites: a literature review. Odontology. 2016 Sep;104(3):257-70.

15. Van Meerbeek B, Peumans M, Poitevin A et al. Relationship between bond-strength tests and clinical outcomes. Dent Mater 2010;26:e100-121.

16. Roulet Marginal integrity: clinical significance. J Dent. 1994;22 Suppl 1:S9-12.

17. Ari H, Dönmez N, Belli S. Effect of artificial saliva contamination on bond strength to pulp chamber dentin. Eur J Dent 2008;2:86-90.

18. Hiraishi $\mathrm{N}$, Kitasako $\mathrm{Y}$, Nikaido $\mathrm{T}$ et al. Effect of artificial saliva contamination on $\mathrm{pH}$ value change and dentin bond strength. Dent Mater 2003;19:429-34.

19. Sattabanasuk V, Shimada Y, Tagami J. Effects of saliva contamination on dentin bond strength using all-in-one adhesives. J Adhes Dent 2006;8:311-8.

20. Silverstone LM, Hicks MJ, Featherstone MJ. Oral fluid contamination of etched enamel surfaces: an SEM study. J Am Dent Assoc 1985;110:329-32.

21. NeelagiriK,Kundabala M, Shashi RA et al. Effects of saliva contamination and decontamination procedures on shear bond strength of self-etch dentine bonding systems: An in vitro study. J Conserv 2010 Apr;13(2):71-5.

22. Elkassas D, Arafa A. Assessment of post-contaminationtreatments affecting different bonding stages to dentin. Eur J Dent.2016 Jul-Sep; 10(3): 327-332.

23. Yoo HM, Oh TS, Pereira PN. Effect of saliva contamination on the microshear bond strength of onestep self-etching adhesive systems to dentin. Oper Dent 2006;31:127-34. 
24. Fritz UB, Finger WJ, Stean H. Salivary contamination during bonding procedures with a one-bottle adhesive system. Quintessence Int 1998;29:567-72.

25. Cobanoglu N, Unlu N, Ozer FF et al. Bond strength of self-etch adhesives after saliva contamination at different application steps. Oper Dent 2013;38:505-11.

\section{Tables}

Table 1: Composition and application mode of the adhesive system and resin-composite used.

Composition

Application mode

\begin{tabular}{|c|c|c|}
\hline $\begin{array}{c}\text { ExciTE F } \\
\text { (Ivoclar } \\
\text { Vivadent, } \\
\text { Schaan, } \\
\text { Liechnstein) }\end{array}$ & $\begin{array}{l}\text { Phosphonic acid acrylate, HEMA, } \\
\text { dimethacrylate, highly dispersed } \\
\text { silicone dioxide, initiators, stabilizers } \\
\text { and potassium fluoride, ethanol }\end{array}$ & $\begin{array}{l}\text { Phosphoric acid gel was applied on dentin for 10-15 } \\
\text { seconds and removed with a vigorous water spray for } \\
\text { at least } 5 \text { seconds. Excess moisture was removed with } \\
\text { an air gun leaving the dentin surface with a glossy wet } \\
\text { appearance (wet bonding). } \\
\text { Dentin was saturated with a generous amount of } \\
\text { ExciTE F for at least } 10 \text { second and excess was } \\
\text { removed with a weak stream of air, leaving a uniform, } \\
\text { glossy appearance. ExciTE F was light-cured for } 10 \\
\text { seconds at } 850 \mathrm{~mW} / \mathrm{cm}^{2} \text {. }\end{array}$ \\
\hline $\begin{array}{c}\text { Resin- } \\
\underline{\text { composite }} \\
\text { Tetric } \\
\text { EvoCeram } \\
\text { (Ivoclar } \\
\text { Vivadent, } \\
\text { Schaan, } \\
\text { Liechnstein) }\end{array}$ & $\begin{array}{l}\text { Bis-GMA, urethane dimethacrylate, } \\
\text { ethoxylated Bis-EMA, barium glass } \\
\text { filler, ytterbiumtrifluoride, mixed } \\
\text { oxide, prepolymers, additives, } \\
\text { catalysts and stabilizers, pigments }\end{array}$ & $\begin{array}{l}\text { Resin-composite was placed in a single layer and } \\
\text { photopolymerized for } 40 \mathrm{~s} \text { at } 850 \mathrm{~mW} / \mathrm{cm}^{2} \text { light } \\
\text { intensity. }\end{array}$ \\
\hline
\end{tabular}

Table 2: Mean values and SD of the \%DM parameter for all groups and depth levels*. 


\begin{tabular}{lcrrr}
\hline Group & & DM-level1 & DM-level2 & DM-level3 \\
\hline Group 1 & Mean & $0.00^{\mathrm{e}, \mathrm{f}}$ & $0.02^{\mathrm{a}, \mathrm{e}}$ & $0.01^{\mathrm{d}, \mathrm{e}}$ \\
(Control) & SD & 0.00 & 0.04 & 0.03 \\
\hline Group 2 & Mean & $0.01^{\mathrm{a}, \mathrm{b}}$ & $0.02^{\mathrm{b}, \mathrm{f}}$ & $0.02^{\mathrm{a}, \mathrm{f}}$ \\
& SD & 0.02 & 0.04 & 0.04 \\
\hline Group 3 & Mean & $0.01^{\mathrm{c}, \mathrm{d}}$ & $0.03^{\mathrm{c}}$ & $0.02^{\mathrm{g} \cdot \mathrm{h}}$ \\
& $\mathrm{SD}$ & 0.03 & 0.06 & 0.04 \\
\hline Group 4 & Mean & $0.05^{\mathrm{a}, \mathrm{c}, \mathrm{e}}$ & $0.06^{\mathrm{a}, \mathrm{b}}$ & $0.08^{\mathrm{b}, \mathrm{d}, \mathrm{f}, \mathrm{g}}$ \\
& $\mathrm{SD}$ & 0.06 & 0.08 & 0.08 \\
\hline Group 5 & Mean & $0.06^{\mathrm{b}, \mathrm{d}, \mathrm{f}}$ & $0.08^{\mathrm{c}, \mathrm{d}, \mathrm{e}, \mathrm{f}}$ & $0.07^{\mathrm{a}, \mathrm{c}, \mathrm{e}, \mathrm{h}}$ \\
& $\mathrm{SD}$ & 0.07 & 0.08 & 0.08 \\
\hline Group 6 & Mean & $0.02^{\mathrm{a}}$ & $0.03^{\mathrm{d}}$ & $0.03^{\mathrm{b}, \mathrm{c}}$ \\
& $\mathrm{SD}$ & 0.04 & $0.05^{2}$ & 0.05 \\
\hline
\end{tabular}

* Same letters within the columns indicate statistically significant differences among the Groups (1 to 6) for each level/column (DM1, DM2, DM3), according to post-hoc analysis, Mann-Whitney $U$ test, $p<0.05$.

Table 3: Mean values and SD of the MG parameter for all groups and depth levels *.

\begin{tabular}{llrrr}
\hline Group & & MG-level1 & MG-level2 & MG-level3 \\
\hline Group 1 & Mean & $0.00^{\mathrm{e}, \mathrm{f}}$ & $5.26^{\mathrm{a}, \mathrm{d}}$ & $3.59^{\mathrm{c}, \mathrm{d}}$ \\
(Control) & SD & 0.00 & 13.06 & 9.42 \\
\hline Group 2 & Mean & $4.49^{\mathrm{a}, \mathrm{b}}$ & $17.05^{\mathrm{e}}$ & 15.40 \\
& SD & 11.65 & 51.81 & 24.91 \\
\hline Group 3 & Mean & $4.92^{\mathrm{c}, \mathrm{d}}$ & $15.31^{\mathrm{b}}$ & $6.68^{\mathrm{e}, \mathrm{f}}$ \\
& SD & 12.51 & 26.89 & 13.95 \\
\hline Group 4 & Mean & $29.43^{\mathrm{a}, \mathrm{c}, \mathrm{e}}$ & $29.89^{\mathrm{a}}$ & $27.89^{\mathrm{a}, \mathrm{c}, \mathrm{e}}$ \\
& SD & 38.86 & 40.44 & 24.88 \\
\hline Group 5 & Mean & $24.10^{\mathrm{b}, \mathrm{d}, \mathrm{f}}$ & $30.74^{\mathrm{b}, \mathrm{c}, \mathrm{d}, \mathrm{e}}$ & $29.34^{\mathrm{b}, \mathrm{d}, \mathrm{f}}$ \\
& SD & 28.17 & 26.81 & 26.39 \\
\hline Group 6 & Mean & 11.70 & $12.07^{\mathrm{c}}$ & $10.52^{\mathrm{a}, \mathrm{b}}$ \\
& SD & 19.11 & 19.97 & 20.32 \\
\hline
\end{tabular}

*Same letters within the columns indicate statistically significant differences among the Groups (1 to 6) for each level/column (MG1, MG2, MG3), according to post-hoc analysis, Mann-Whitney $U$ test, $p<0.05$. 
Figures

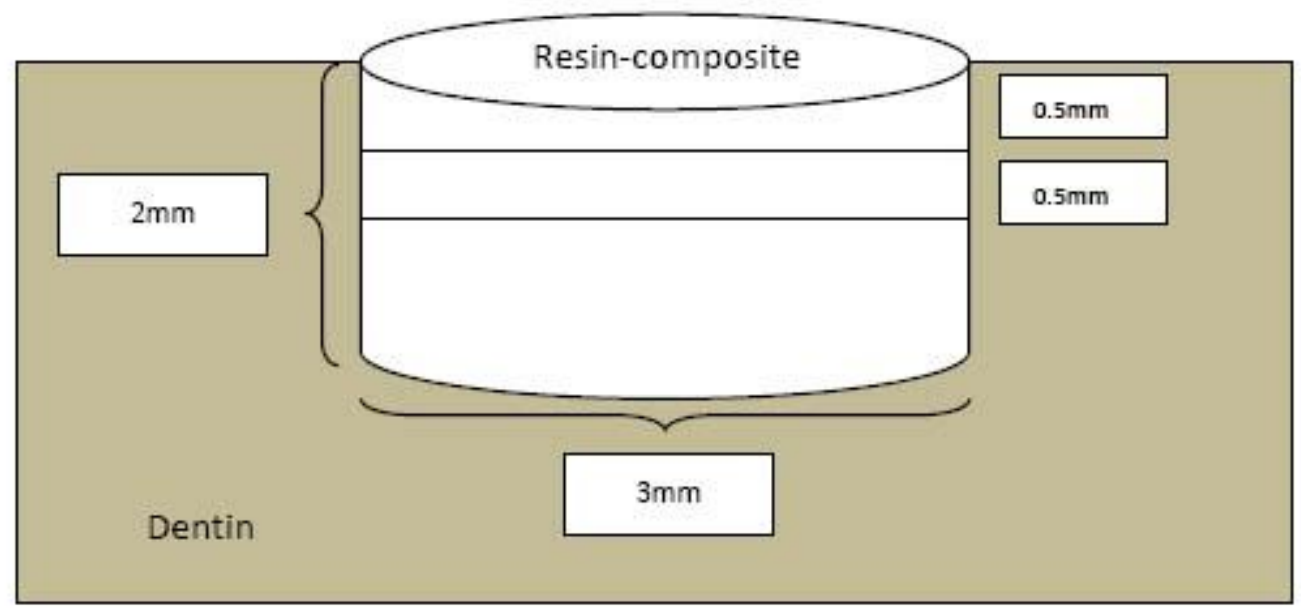

\section{Figure 1}

Schematic cross-sectional view of the specimen. Wall adaptation was evaluated at the upper surface and at two consecutive depth levels of $0.5 \mathrm{~mm}$ each, after gradual grinding of each sample.

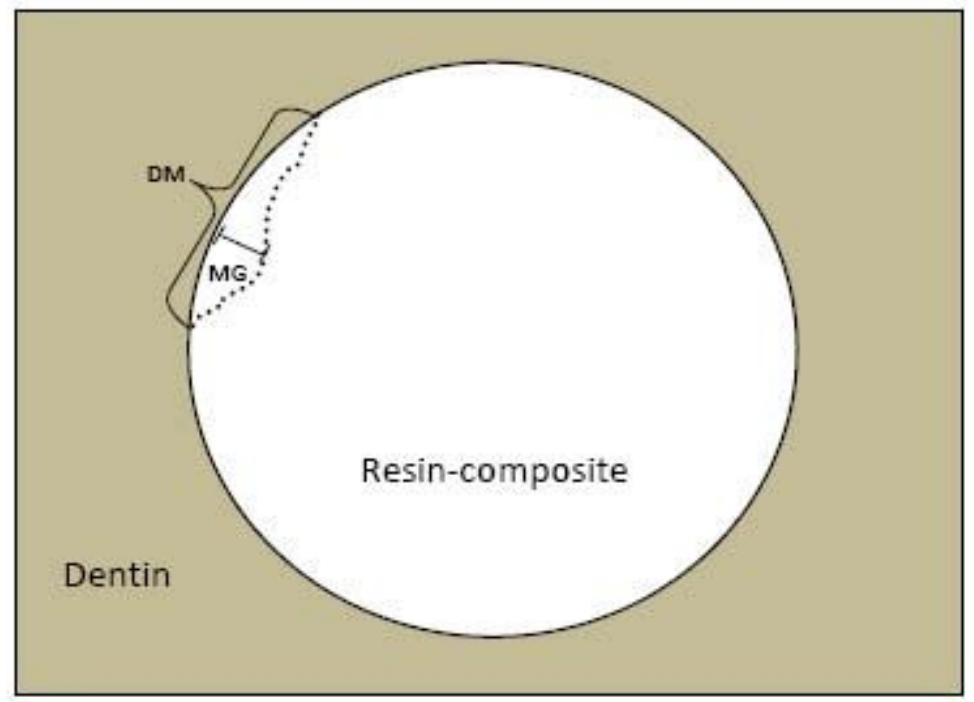

\section{Figure 2}

Schematic occlusal view of the specimen. Marginal and internal adaptation was evaluated by measuring the length of the debonded margins (DM) relative to the cavity periphery (\%DM) and the width of the maximum marginal gap (MG). 


\section{Resin- composite}

\section{Figure 3}

Restoration with perfect marginal adaptation 


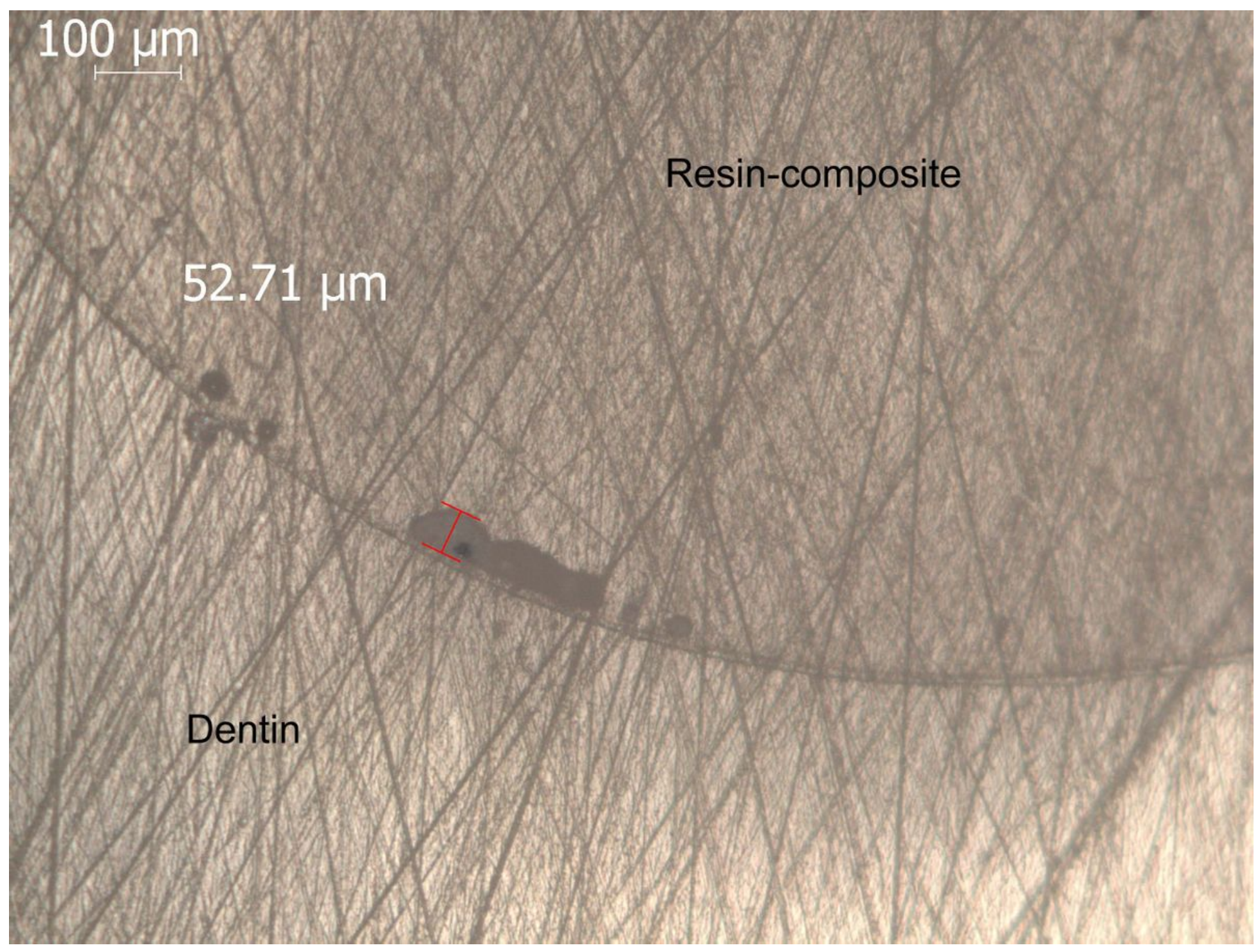

Figure 4

Restoration with debonded margins 